\title{
Wind-Photovoltaic-Storage Hybrid System Coordinated Optimal Control of Output Power
}

\author{
Hao Tian ${ }^{1, a}$, Yutian Liu ${ }^{1, b}$ \\ ${ }^{1}$ Key Laboratory of Power System Intelligent Dispatch and Control of Ministry of Education, \\ Shandong University, Jinan 250061, China \\ atian1992hao@126.com, bliuyt@sdu.edu.com
}

Keywords: Wind-photovoltaic-storage hybrid system; Multi-objective optimization; Non-dominated sorting genetic algorithm (NSGA-II); Evaluation method.

\begin{abstract}
For large wind-photovoltaic-storage hybrid system, in order to maximize the match between output of the hybrid system and the scheduling plan, it is very important to coordinate the output power of wind, photovoltaic and energy storage system. Taking the uncertain of wind, photovoltaic generation and the power ramp constraint into account, a coordinated optimal control strategy is proposed. The aim of optimization model is to minimize the average expectation of power deviation and charge-discharge times of battery and maximize the average residual capacity of battery. The Non-dominated sorting genetic algorithm (NSGA-II) is used to solve the multi-objective problem for getting the alternative schemes set, and an evaluation method is given to acquire the best scheme. The feasibility of the present strategy is examined by simulation.
\end{abstract}

\section{Introduction}

The wind-photovoltaic-storage hybrid system, which takes complementary feature between wind and photovoltaic generation into consideration, can reduce the fluctuation of output power and enhance the ability for distributed generators to connect to the grid [1]. Recently, several researches about wind-photovoltaic-storage hybrid system have been published. Literature [2] addressed optimal control of energy storage based on fuzzy correlated-chance programming. The final goal is to maximize the mean value of the total credibility by using the fuzzy simulation. Literature [3] proposed an optimal control of battery energy storage system based on variable smoothing time constant. Literature [4] put forward the sectionalization gradation strategy for battery, which could track the power change of wind power and photovoltaic generation.

In this paper, the coordinated day-ahead optimal control strategy is addressed which takes the uncertain of wind, photovoltaic generation and the power ramp constraint into account. The aim of the multi-objective control strategy is to minimize the average math expectation of power deviation and charge-discharge times of battery station and maximize the average residual capacity of battery. By using non-dominated sorting genetic algorithm (NSGA-II) and evaluation method to solve the model, the specific output power strategy is given for wind generators, photovoltaic generators and battery storage individually in each period.

\section{Coordinated Optimal Control of Output Power}

Firstly one day into is divided 24 periods, coordinate the output power of wind, photovoltaic generators and storage system in every period to satisfy the scheduling curve. Choosing the appropriate mathematical model to describe the uncertain of wind power and photovoltaic, establish the objective functions and constraint in mathematical expressions. Then use NSGA-II algorithm and evaluate method to solve the model.

Forecast error of wind-photovoltaic in math expression. At present, wind power and photovoltaic have relatively large error in the forecast mainly due to the error from numerical weather 
forecast, model of output power and prediction method [5]. The forecast error rate could be shown in(1)

$$
\varepsilon=\frac{P_{\text {real }}-P_{\text {forcast }}}{P_{\text {forcast }}}
$$

Where $\varepsilon$ is the forecast error rate, stand for the percentage of forecast error; $\mathrm{P}_{\text {real }}$ and $\mathrm{P}_{\text {forcast }}$ are the real output and forecast output respectively.

According to recent research about error distribution, Beta distribution, Normal distribution, Cauchy distribution and Laplace distribution have been applied to describ the model [6]. Each distribution has it own advantages and scope of application. In this paper, the forecast error rate is defined to obeied to Normal distribution, which shown in(2):

$$
f\left(x ; \mu, \sigma^{2}\right)=\frac{1}{\sqrt{2 \pi \sigma^{2}}} e^{-\frac{(x-\mu)^{2}}{2 \sigma^{2}}}
$$

Where $\mu$ is the mean or expectation of the distribution and the parameter $\sigma$ is its standard deviation.

Objective function. The main goal of coordinated optimal control is to improve the ability to track scheduling plan and reduce the compensation pressure of battery. Comprehensive analyzing of the above situation, optimization model will help to minimize the average math expectation of power deviation and charge-discharge times of battery station and maximize the average residual capacity of battery station [7]. It is a typical multi-objective problem.

(1) Objective function one: Minimize the average expectation of power deviation. The forecast error distribution is obeyed to Normal distribution, and then dividing the probability distribution function into several sections, caculate the integration area of each section. Take equation (3)(4)(5) to get the average expectation of power deviation.

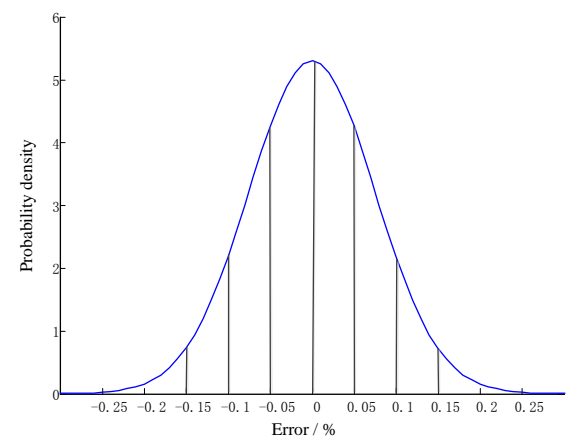

Fig. 1 Dividing the probability distribution function

$$
\begin{aligned}
& \min f_{1}(x)=\min \frac{1}{N} \sum_{i=1}^{N} E(i) \\
& E(i)=\sum_{j=1}^{M} P[a(j)<\varepsilon<b(j)] \cdot e(i, j) \\
& e(i, j)=\left[P_{\text {wind }}(i, j)+P_{p v}(i, j)+P_{s o c}(i, j)-P_{o b j}(i)\right] / P_{o b j}(i)
\end{aligned}
$$

Where $\mathrm{i}$ is the time period, $\mathrm{i}=1,2, \cdots \mathrm{N}$; j reprsents the section of probability distribution function, $j=1,2, \cdots M$; e(i,j) reprsents the power deviation in No.i period and No.j section. $P_{\text {wind }}(i, j), P_{\text {solar }}(i, j)$ are the real output of wind generators and photovoltaic generators in No.i period and No.j section, $P_{\text {soc }}(i, j)$ is the charge-discharge power in No.i period and No.j section, $P_{\text {soc }}(i, j)>0$ means discharge, $\mathrm{P}_{\text {soc }}(\mathrm{i}, \mathrm{j})<0$ means charge; $\mathrm{P}_{\text {obj }}$ is the schedule output.

(2) Objective function two: Minimize the charge-discharge times of battery. The battery storage has three statuses in normal operating state, which are charging, discharging and shutdown. The charge-discharge times will increase one when the status change.

$\min f_{2}(x)=\min N_{b}$ 
(3) Objective function three: Maximize the average residual capacity of battery station. It is helpful to enhance the adjustment ability for next control period in case the emergency happens which could make power shortfall.

$$
\max f_{3}(x)=\max \frac{1}{N} \sum_{i=1}^{N} E_{s o c}(i)
$$

Where $E_{\text {soc }}(i)$ is residual capacity after No. i period. $E_{\text {soc }}(i)=1$ means full of power and $E_{\text {soc }}(i)=0$ means out of power.

\section{Constraints.}

(1) The output power and charge or discharge depth of battery will satisfy the constraints because the battery has limitation from physical characteristics [8].

$$
\left\{\begin{array}{l}
E_{\min } \leq E_{s o c}(i) \leq E_{\max } \\
-P_{c h \_\max } \leq P_{\text {soc }}(i) \leq P_{\text {dic_max }}
\end{array}\right.
$$

Where $\mathrm{E}_{\min }$ is the minimum of residual capacity; $\mathrm{E}_{\max }$ is the maximum of residual capacity; $\mathrm{P}_{\text {ch-max }}$ is the largest power of charging; $\mathrm{P}_{\mathrm{dic}-\max }$ is the largest power of discharging.

The recursion formula of residual capacity in each period is:

$$
\begin{cases}E_{s o c}(i)=E_{s o c}(i-1)(1-\delta)+P_{s o c}(i) \cdot \eta_{C} / S_{\text {total }} & P_{s o c}(i)>0 \\ E_{s o c}(i)=E_{s o c}(i-1)(1-\delta)+P_{s o c}(i) /\left(\eta_{D} \cdot S_{\text {total }}\right) & P_{s o c}(i)>0\end{cases}
$$

Where $\delta$ is the self-discharge rate of battery; $\mathrm{E}_{\text {soc }}(\mathrm{i}-1)$ is the residual capacity of $\mathrm{i}-1$ period; $\eta_{\mathrm{C}}$ is the discharging efficiency; $\eta_{D}$ is the charging effciency.

(2) The output of wind generators and photovoltaic generators has constraints because of the speed of power ramp. In order to simplify the calculations, only consider the speed of up-ramping and ignore the down-ramping.

$$
\begin{aligned}
& \left\{\begin{array}{l}
0<P_{\text {wind }}(i, j)<[1+\varepsilon(i, j)] \cdot P_{\text {wind }} \text { forc } \\
V_{\text {wind }}(i, j)<V_{\text {wind_max }}
\end{array}\right. \\
& \left\{\begin{array}{l}
0<P_{p v}(i, j)<[1+\varepsilon(i, j)] \cdot P_{p v_{-} \text {forc }} \\
V_{p v}(i, j)<V_{p v_{-} \max }
\end{array}\right.
\end{aligned}
$$

Where $\mathrm{P}_{\mathrm{pv} \_ \text {forc }}$ and $\mathrm{P}_{\text {wind_forc }}$ are the forecast output power of photovoltaic and wind generators. $\varepsilon(\mathrm{i}, \mathrm{j})$ is the prediction error rate of No.i period and No.j section. $V_{p v}(i, j)$ and $V_{\text {wind }}(i, j)$ are the up-ramping speed of photovoltaic and wind generators in No.i period and No.j section, $\mathrm{V}_{\mathrm{pv} \text {-max }}$ and $\mathrm{V}_{\mathrm{pv} \text {-min }}$ are the the maximum limit of speed of photovoltaic and wind generators.

\section{Solving Method and Scheme Selection}

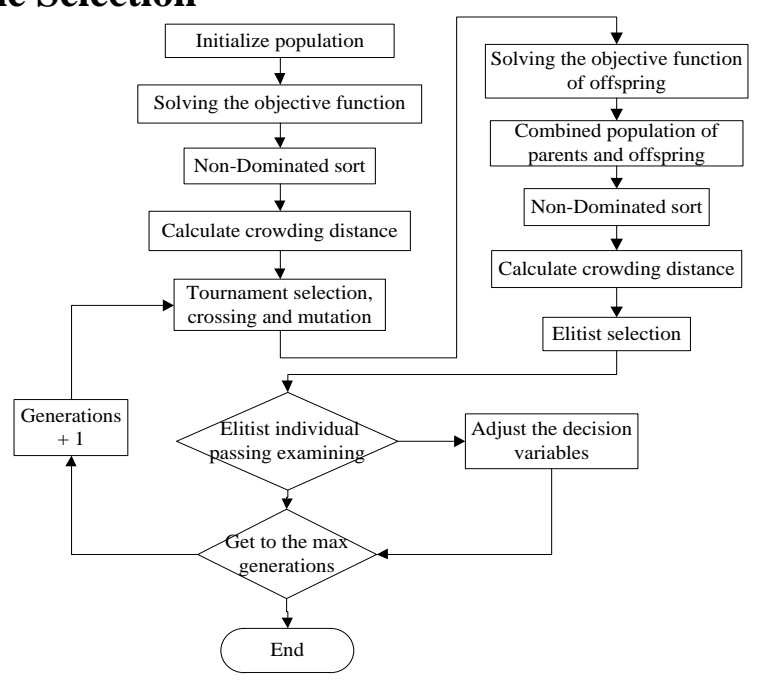

Fig. 2 Flowchart of NSGA-II algorithm 
NSGA-II is a popular non-domination based genetic algorithm for multi-objective optimization. It is a global optimization algorithm which could obtain the pareto front in a short time. In this paper, we regard the output power of wind, photovoltaic and storage as decision variables, the solution flowchart is shown in figure 2.

After using NSGA-II algorithm, the optimal solution set is obtained. Then an evaluation method is adopted and convert three objectives into evaluation indexes, determine the final scheme by relative comparison method [9].

During the multiple indexes decision making, each evaluation index has different unit, dimensional and magnitude which could affect the scheme selection. So it is essential to unify the raw criteria data into same standard. The three evaluation indexes are average expectation of power deviation $\mathrm{f}_{\Delta \mathrm{P}}(\mathrm{i})$, charge-discharge times of battery storage $\mathrm{f}_{\mathrm{N}}(\mathrm{i})$, average residual capacity of battery storage $f_{\text {soc }}(i)$. For evaluation index $f_{\Delta P}(i)$ and $f_{N}(i)$, smaller is better. But for evaluation index $f_{\text {soc }}(i)$, the bigger the better. The dealing approach is shown in(12):

$$
\left\{\begin{array}{l}
\hat{f}_{\Delta P}=\frac{\max f_{\Delta P}-f_{\Delta P}(i)}{\max f_{\Delta P}-\min f_{\Delta P}} \\
\hat{f}_{N}=\frac{\max f_{N}-f_{N}(i)}{\max f_{N}-\min f_{N}} \\
\hat{f}_{s o c}=\frac{f_{\Delta P}(i)-\min f_{\Delta P}}{\max f_{\Delta P}-\min f_{\Delta P}}
\end{array}\right.
$$

Where, min and max are the minimum and maximum of evaluation index individually. After transformation, three evaluation indexes are all monotonic and in the range of [0,1]. In decision-making analysis, it is normal to use linear weighting to judge the schemes.

$$
S(i)=\lambda_{1} \hat{f}_{\Delta P}+\lambda_{2} \hat{f}_{N}+\lambda_{3} \hat{f}_{s o c}
$$

Where $\lambda_{1}, \lambda_{2}, \lambda_{3}$ represent the weighting coefficient which satisfy the constraint $\lambda_{1}+\lambda_{2}+\lambda_{3}=1$.

It is crucial to decide the weighting coefficients which determine the correctness of this evaluation method. The deciding method including expert method, the binomial coefficient weighted method and relative comparison method. We utilize relative comparison method to decide the weighting coefficient, and this method just need decision makers to judge the importance between two of this evaluation indexes. The calculation equation is shown in(14):

$$
\lambda_{i}=\frac{\sum_{j=0}^{n} \lambda_{i j}}{\sum_{j=0}^{n} \sum_{i=0}^{n} \lambda_{i j}}
$$

Where $\lambda_{\mathrm{ij}}+\lambda_{\mathrm{ji}}=1(\mathrm{i} \neq 0), \lambda_{\mathrm{ii}}=0$.

For the three evaluation indexes $\left\{\mathrm{f}_{\Delta \mathrm{P}}, \mathrm{f}_{\mathrm{N}}, \mathrm{f}_{\mathrm{soc}}\right\}$, based on engineering practice, the relative relationship between them are: $f_{N}>f_{\Delta P}, f_{N}>f_{s o c}, f_{\Delta P}>f_{\text {soc }}$. Table 1 gives the relative importance evaluation of index.

Table 1 Relative importance evaluation of index

\begin{tabular}{cccc}
\hline Index & $\mathrm{f}_{\Delta \mathrm{P}}$ & $\mathrm{f}_{\mathrm{N}}$ & $\mathrm{f}_{\mathrm{soc}}$ \\
\hline $\mathrm{f}_{\Delta \mathrm{P}}$ & 0 & 0.2 & 0.6 \\
$\mathrm{f}_{\mathrm{N}}$ & 0.8 & 0 & 0.8 \\
$\mathrm{f}_{\mathrm{soc}}$ & 0.4 & 0.2 & 0 \\
\hline
\end{tabular}

According to the relative importance evaluation in Table 1, the selection strategy is shown in(15). The scheme with the biggest $\mathrm{S}$ will be the final optimal control scheme.

$$
S(i)=0.267 \hat{f}_{\Delta P}+0.533 \hat{f}_{N}+0.2 \hat{f}_{s o c}
$$




\section{Simulation}

The scales of the following case study refer to a real wind-photovoltaic-storage project in China. It includes 500MW wind generators, $100 \mathrm{MW}$ photovoltaic generators and $110 \mathrm{MW} / 440 \mathrm{MW} \cdot \mathrm{h}$ storage battery. The up-ramping speed of wind generators and photovoltaic generators are $20 \mathrm{MW} \cdot \mathrm{min}$ and $5 \mathrm{MW} \cdot \mathrm{min}$ individually. The self-discharge rate of storage battery $\delta=0$, charge efficiency $\gamma_{\mathrm{C}}$ and $\gamma_{\mathrm{D}}$ are both $1, E_{\max }=0.9, E_{\min }=0.1$, initial battery capacity $E_{\text {soc }}=0.5$ 。 The prediction error of wind and photovoltaic is given by history data, $\mu=0, \sigma=0.2744$. The sections of error distributed function are shown in Table 2.

Table 2 The integration of each part of distributed function

\begin{tabular}{cccc}
\hline $\mathrm{P}(\varepsilon \leq-0.15)$ & $\mathrm{P}(-0.15<\varepsilon \leq-0.1)$ & $\mathrm{P}(-0.1<\varepsilon \leq-0.05)$ & $\mathrm{P}(-0.05<\varepsilon \leq 0)$ \\
\hline 0.0232 & 0.0689 & 0.1612 & 0.2467 \\
\hline $\mathrm{P}(0<\varepsilon \leq 0.05)$ & $\mathrm{P}(0.05<\varepsilon \leq 0.1)$ & $\mathrm{P}(0.1<\varepsilon \leq 0.15))$ & $\mathrm{P}(\varepsilon>0.15)$ \\
\hline 0.2467 & 0.1612 & 0.0689 & 0.0232 \\
\hline
\end{tabular}

The predicted output of wind generation and photovoltaic generation is shown in Figure 3. The predicted output of wind-photovoltaic hybrid system and generation schedule is shown in Figure 4.

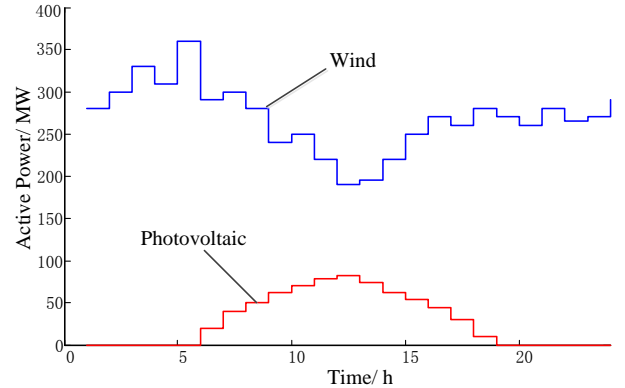

Fig. 3 Prediction curve of wind and photovoltaic

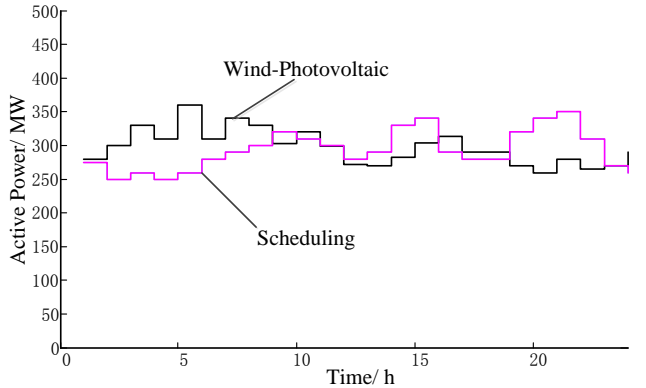

Fig. 4 Wind-photovoltaic output and scheduling

The parameters of NSGA-II algorithm are: population 100, generation 500, the distribution indices for crossover and mutation are both 20 . The simulation result is shown below:

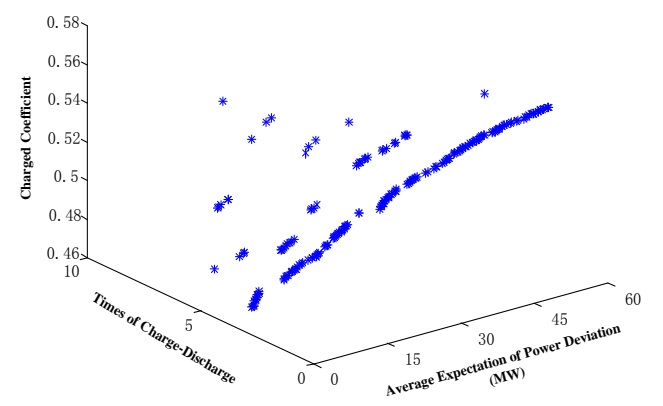

Fig. 5 Simulation result of NSGA-II algorithm

Base on the simulation result of NSGA-II algorithm, equation(15) is used to find the best scheme. The chosen generation control scheme is shown in Figure 6, 7. It could be seen that the hybrid system can match the scheduling curve perfectly and the average expectation of power deviation is nearly 30MW. The charge-discharge times of storage battery are 3, which reduce the frequency of charge-discharge and ensure the lifetime of battery.



Fig. 6 The control scheme of hybrid system

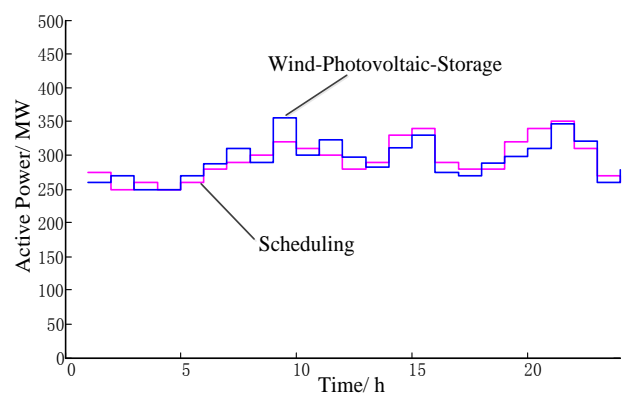

Fig. 7 The control scheme and scheduling curve 


\section{Summary}

In this paper, a coordinated optimal control strategy is proposed for wind-photovoltaic-storage hybrid system. Taking the prediction error of wind, photovoltaic generation and the power ramp constraint into account, the target of optimization model is to minimize the average math expectation of power deviation and charge-discharge times of battery station and maximize the average residual capacity of battery station. After establishing the mathematical model, the NSGA-II algorithm is adopted to find the optimal solution set, and use evaluating method to get the final scheme. The feasibility of the present strategy is examined through simulation. In the meantime, several shortages in the study need to be improved. (1) The 24 periods in one day are inadequate to describe the output feature of system specifically.(2) The day-ahead control strategy is provided for the wind-photovoltaic-storage hybrid system which lasting a long time and may cause large error compare with the real operation system. The next stage of study will focus on 96 periods one day and propose the real-time optimal strategy.

\section{Acknowledgments}

This work is supported by National Basic Research Program of China. (973 Program) (No.2012CB215101)

\section{References}

[1] Nerea Ruiz, Iñigo Cobelo, and José Oyarzabal. “A Direct Load Control Model for Virtual Power Plant Management,” IEEE Trans Power Syst, 2009; 24(2):959 -966.

[2] Yongqiang HU, Chenliang LIU, Shuqiang ZHAO, Mingyu WANG. “Optimal Control of Energy Storage Based on Fuzzy Correlated-chance Programming," Automation of Electric Power Systems, 2014; 38(6):20 -24.

[3] lunwen XIE, Jiming LU, Chengxiong MAO, Dan WANG, Xueqiang FU, Wusheng LIN. "Optimal Control of Battery Energy Storage System Based on Variable Smoothing Time Constant," Automation of Electric Power Systems, 2013; 37(1):96 -102.

[4] Jinxin Li, Jiancheng Zhang. "Optimization method to fluctuations of predictive power of HPWS”. Power Sources Technology, 2013; 37(1):62 -64.

[5] SODER L. Reserve margin planning in a wind-hydro-thermal power system. IEEE Trans Power Syst, 1993, 8(2): 1345-1354.

[6] Fang LIU, Yi PAN, Hui LIU, Qian DING, Qiang LI, Zhiming WANG. Piecewise Exponential Distribution Model of Wind Power Forecasting Error. Automation of Electric Power Systems, 2013, 37(18): 14-19.

[7] Yongzhi Qi, Yutian Liu. Output Power Rolling Optimization and Real-Time Control in Wind-Photovoltaic-Storage Hybrid System. Transactions of China Electrotechnical Society, 2014; 29(8):265 -273.

[8] Ming DING, Ningzhou XU, Rui BI. Modeling of BESS for smoothing renewable energy output fluctuation. Automation of Electric Power Systems, 2011, 35(2):66-72.

[9] Xiaojiang GUO, Jianbo GUO, Shiying MA, Chengshan WANG, Yantao ZHANG. A Method for Multi DC Terminal Location Selection Based on Multi-infeed Short Circuit Ratio. Proceedings of the CSEE, 2013; 33(10):36 -42. 